\title{
Self-Diffusion of Particles Interacting Through a Square-Well or Square-Shoulder Potential
}

\author{
Harald Wilbertz, ${ }^{1,2}$ Jan Michels, ${ }^{3}$ Henk van Beijeren, ${ }^{4}$ \\ and Jan Adriaan Leegwater ${ }^{4}$
}

Received June 10, 1988

\begin{abstract}
The diffusion coefficient and velocity autocorrelation function for a fluid of particles interacting through a square-well or square-shoulder potential are calculated from a kinetic theory similar to the Davis-Rice-Sengers theory and the results are compared to those of computer simulations. At low densities the theory yields too low estimates due to the neglect of correlations between subsequent partial collisions of identical pairs; in particular, the neglect of boundstate effects appears important. At intermediate densities the theory makes reasonable predictions and. at high densities it produces too high values, due to the neglect of ring terms and other correlated collision events. The results for the square-shoulder potential generally exhibit better agreement between theory and simulations than do those for the square-well potential.
\end{abstract}

KEY WORDS: Square-well potential; diffusion coefficient; kinetic theory; velocity autocorrelation function; computer simulations.

\section{WHY STUDY A SOUARE-WELL FLUID?}

Since the celebrated paper of Alder and Wainwright, ${ }^{(1)}$ many investigations based on the method of molecular dynamics (MD) simulations have been done to study the transport properties of dense classical fluids. Most of them studied either systems of hard spheres or liquids consisting of particles interacting through a Lennard-Jones ( $\mathrm{LJ}$ ) potential. The hard-sphere

\footnotetext{
${ }^{1}$ Institut für Theoretische Physik, Rheinisch Westfälische Technische Hochschule, Federal Republic of Germany.

${ }^{2}$ Present address: AEG, Geschäftsbereich Informationstechnik, Konstanz, Federal Republic of Germany.

${ }^{3}$ Van der Waals Laboratorium, 1018 XE Amsterdam, The Netherlands.

${ }^{4}$ Instituut voor Theoretische Fysica, 3508 TA Utrecht, The Netherlands.
} 
simulations can be compared to the predictions of Enskog's theory ${ }^{(2)}$ and more recent refinements ${ }^{(3)}$ of this, and up to fairly high densities good agreement has been found. ${ }^{(4)}$ Due to the complications caused by the finite duration of collisions and the presence of many-body interactions, no comparable theory is available for dense LJ systems, although good agreement is found between MD results for these and experimental results for noble gases. $^{(5)}$ If one wants to extend kinetic theory from hard-sphere fluids to more realistic potentials, containing attractions between particles and allowing for a nontrivial temperature dependence of transport coefficients, the square-well potential is a most natural choice. Since also the interactions at the outer square-well edge are instantaneous, the problems referred to above are avoided.

An extension of Enskog's theory to the square-well potential, largely based on intuitive ideas, was presented by Davis et al. (DRS) ${ }^{(6)}$ but their theory is not entirely satisfactory, because conservation of energy is not obeyed. This problem is avoided in a recent derivation of a kinetic theory for the square-well fluid, ${ }^{(7)}$ employing maximization of entropy as a formal tool. The resulting hydrodynamic equations and transport coefficients, for a simple fluid, up to the Navier-Stokes level are almost the same as those obtained from DRS theory; only the bulk viscosity is different. ${ }^{(26)}$ Yet, already the fulfilment of all conservation laws renders this new theory more satisfactory.

In this paper we consider the related kinetic equation describing tagged particle motion in a square-well fluid at equilibrium and compare its predictions for the coefficient of self-diffusion and for the velocity autocorrelation function to the results of the MD simulations.

The organization of the paper is as follows: in Section 2 we introduce the kinetic equation for the square-well fluid and derive from it the equation describing tagged particle motion; in Section 3 we discuss the calculation of the self-diffusion coefficient; in Section 4 we compare theoretical and MD values of this coefficient; in Section 5 we consider our theoretical predictions and MD simulation results for the velocity autocorrelation function and in Section 6 we discuss the results obtained.

\section{KINETIC EQUATION FOR A DENSE SQUARE-WELL FLUID}

We consider identical particles of mass $m$ with phases $x_{i}=\left(\mathbf{r}_{i}, \mathbf{v}_{i}\right)$, where $\mathbf{r}_{i}$ is the position and $\mathbf{v}_{i}$ the velocity of particle $i$. They interact through a square-well potential $\varphi\left(r_{i j}\right)$, equal to $\infty$ for $r_{i j}<\sigma$, to $-\varepsilon \equiv-m v_{l}^{2} / 4$ for $\sigma \leqslant r_{i j}<R$, and to 0 for $r_{i j} \geqslant R$, with $r_{i j}=\left|\mathbf{r}_{i j}\right|$ and $\mathbf{r}_{i j}=$ $\mathbf{r}_{i}-\mathbf{r}_{j}$. The parameter $\varepsilon$ can also take negative values, in which case we refer to the square-shoulder potential. The time evolution of the one-particle 
distribution function $f_{1}(x, t)$ and that of the local potential energy density $u(\mathbf{r}, t)$ are determined, respectively, by

$$
\begin{gathered}
\left(\frac{\partial}{\partial t}+\mathbf{v}_{1} \cdot \frac{\partial}{\partial \mathbf{r}_{1}}\right) f_{1}\left(x_{1}, t\right)=\int d x_{2} \bar{T}_{12} f_{2}\left(x_{1}, x_{2}, t\right) \\
\frac{\partial}{\partial t} u\left(\mathbf{r}_{1}, t\right)+\frac{1}{2} \frac{\partial}{\partial \mathbf{r}_{1}} \cdot \int d \mathbf{v}_{1} \int d x_{2} \mathbf{v}_{1} \phi\left(r_{12}\right) f_{2}\left(x_{1}, x_{2}, t\right) \\
=\frac{\varepsilon}{2} \int d \mathbf{v}_{1} \int d x_{2}\left|\mathbf{v}_{12} \cdot \hat{\boldsymbol{\sigma}}_{12}\right| f_{2}\left(x_{1}, x_{2}, t\right)\left[\theta\left(\mathbf{v}_{12} \cdot \hat{\boldsymbol{\sigma}}_{12}-v_{l}\right)\right. \\
\left.\times \delta\left(r_{12}-R^{-}\right)-\theta\left(-\mathbf{v}_{12} \cdot \hat{\boldsymbol{\sigma}}_{12}\right) \delta\left(r_{12}-R^{+}\right)\right]
\end{gathered}
$$

with the following definitions:

$$
\begin{aligned}
f_{1}(x, t) & =\left\langle\sum_{i} \delta\left(x-x_{i}\right)\right\rangle \\
u\left(\mathbf{r}_{1}, t\right) & =\frac{1}{2}\left\langle\sum_{i \neq j} \varphi\left(r_{i j}\right) \delta\left(\mathbf{r}_{i}-\mathbf{r}_{1}\right)\right\rangle \\
f_{2}\left(x, x^{\prime}, t\right) & =\left\langle\sum_{i \neq j} \delta\left(x-x_{i}\right) \delta\left(x^{\prime}-x_{j}\right)\right\rangle \\
\langle(\cdots)\rangle & =\sum_{N=0}^{\infty} \int d x^{N} \rho\left(x^{N}, t\right)(\cdots)
\end{aligned}
$$

where $\rho\left(x^{N}, t\right)$ is the density in phase space.

The time evolution equations can be closed by approximating $\rho\left(x^{N}, t\right)$ with the ensemble $\rho_{M}\left(x^{N}, t\right)$ that maximizes a certain entropy functional under the constraints that, at given $t$, both $f_{1}$ and $u$ are reproduced correctly by $\rho_{M}$. Details of this procedure are given in ref. 7; for our discussion the main results are that the velocities of the particles are uncorrelated, and hence

$$
f_{2}\left(x_{1}, x_{2}, t\right)=f_{1}\left(x_{1}, t\right) f_{1}\left(x_{2}, t\right) g_{2}\left(\mathbf{r}_{1}, \mathbf{r}_{2}, t\right)
$$

and $g_{2}\left(\mathbf{r}_{1}, \mathbf{r}_{2}, t\right)$ is a known functional of number density and potential energy temperature at all positions in the fluid.

The number density is determined as $n\left(\mathbf{r}_{1}, t\right)=\int d \mathbf{v}_{1} f_{1}\left(x_{1}, t\right)$ and potential energy temperature is determined by the constraint ${ }^{(7)}$ that the ensemble maximizing entropy correctly describes the local internal energy density as given through $f_{1}\left(x_{1}, t\right)$ and $u\left(\mathbf{r}_{1}, t\right)$. 
The binary collision operator can be defined ${ }^{(7)}$ through the action of its adjoint operator $T_{i j}$ satisfying

$$
\int d \mathbf{v}_{i} d \mathbf{v}_{j} f^{*} T_{i j} g=\left(\int d \mathbf{v}_{i} d \mathbf{v}_{j} g^{*} \bar{T}_{i j} f\right)^{*}
$$

for any pair $f, g$ of square integrable functions of $\mathbf{v}_{i}$ and $\mathbf{v}_{j}$. Four different terms in $T_{i j}=\sum_{l=1}^{4} T_{i j}^{(l)}$ respectively describe collisions between particles $i$ and $j$ at the hard core $(l=1)$, entering $(l=2)$ or leaving $(l=3)$ the well, and rebounding at the inner side of the square-well edge $(l=4)$. Explicitly,

$$
T_{i j}^{(l)}=\lim _{t \rightarrow 0+} S_{t}^{0} \delta\left(r_{i j}-R_{l}\right)\left|\mathbf{v}_{i j} \cdot \mathbf{r}_{i j}\right| \theta_{l}\left(\mathbf{v}_{i j} \cdot \hat{\mathbf{r}}_{i j}\right)\left[b^{(l)}\left(\hat{r}_{i j}\right)-1\right] S_{t}^{0}
$$

where

$$
\begin{gathered}
R_{1}=\sigma, \quad R_{2}=R_{3}=R_{4}=R \\
\mathbf{v}_{i j}=\mathbf{v}_{i}-\mathbf{v}_{j}, \quad \hat{\mathbf{r}}_{i j}=\mathbf{r}_{i j} /\left|\mathbf{r}_{i j}\right| \\
\theta_{1}(x)=\theta_{2}(x)=\theta(-x), \quad \theta_{3}(x)=\theta\left(x-v_{l}\right), \quad \theta_{4}(x)=\theta(x) \theta\left(v_{l}-x\right)
\end{gathered}
$$

and $\theta(x)$ denotes the unit step function.

The collision operators $b^{(l)}(\hat{\boldsymbol{\sigma}})$ change the particle velocities according to the collision dynamics. The center-of-mass velocity $\left(v_{i}+v_{j}\right) / 2$ is unchanged, while the action on the relative velocity is given by

$$
\begin{aligned}
b^{(1)}(\hat{\boldsymbol{\sigma}}) \mathbf{v}_{i j} & =b^{(4)}(\hat{\boldsymbol{\sigma}}) \mathbf{v}_{i j}=\mathbf{v}_{i j}-2 \hat{\boldsymbol{\sigma}}\left(\mathbf{v}_{i j} \cdot \hat{\boldsymbol{\sigma}}\right) \\
b^{(2)}(\hat{\boldsymbol{\sigma}}) & =\mathbf{v}_{i j}-\hat{\boldsymbol{\sigma}}\left\{\left[\left(\mathbf{v}_{i j} \cdot \hat{\boldsymbol{\sigma}}\right)^{2}+v_{l}^{2}\right]^{1 / 2}+\mathbf{v}_{i j} \cdot \hat{\boldsymbol{\sigma}}\right\} \\
b^{(3)}(\hat{\boldsymbol{\sigma}}) & =\mathbf{v}_{i j}+\hat{\boldsymbol{\sigma}}\left\{\left[\left(\mathbf{v}_{i j} \cdot \hat{\boldsymbol{\sigma}}\right)^{2}-v_{l}^{2}\right]^{1 / 2}-\mathbf{v}_{i j} \cdot \hat{\boldsymbol{\sigma}}\right\}
\end{aligned}
$$

The free streaming operators $S_{t}^{0}$ are inserted to produce unequivocal results when $T_{i j}^{(l)}$ acts upon functions that are discontinuous at $r_{i j}=R_{l}$.

From (2.1), (2.7) a kinetic equation for tagged particle motion is obtained by assuming that one single particle, which can be distinguished from the others, has to be described by a nonequilibrium distribution function, whereas for the other particles, with which the tagged particle makes collisions, the equilibrium distribution is adequate at all times. This amounts to replacing $f_{2}\left(x_{1}, x_{2}, t\right)$ in (2.7) by $f_{1}\left(x_{1}, t\right) n \varphi^{\mathrm{eq}}\left(v_{2}\right) g_{2}\left(r_{12}\right)$, with $\varphi^{\mathrm{cq}}(v)$ the Maxwellian velocity distribution

$$
\varphi^{\mathrm{eq}}(v)=(\beta m / 2 \pi)^{3 / 2} \exp \left(-\beta m v^{2} / 2\right)
$$

where $\beta=1 / k_{\mathrm{B}} T, T$ is the temperature, $k_{\mathrm{B}}$ is Boltzmann's constant, and $g_{2}\left(r_{12}\right)$ is the equilibrium pair correlation function at the given density $n$ 
and temperature $T$. The equation for $f_{1}\left(x_{1}, t\right)$ thereby decouples from the energy equation (2.2) and assumes the form

$$
\begin{aligned}
\left(\frac{\partial}{\partial t}+\mathbf{v}_{1} \cdot \frac{\partial}{\partial \mathbf{r}_{1}}\right) f_{1}\left(x_{1}, t\right) & =\int d x_{2} \bar{T}_{12} g_{2}\left(r_{12}\right) n \varphi^{\mathrm{eq}}\left(v_{2}\right) f_{1}\left(x_{1}, t\right) \\
& \equiv: \Lambda f_{1}\left(x_{1}, t\right)
\end{aligned}
$$

which defines the Lorentz-Boltzmann operator $A$.

\section{CALCULATION OF THE COEFFICIENT OF SELF-DIFFUSION}

Self-diffusion is the macroscopic transport phenomenon that describes the motion of tagged molecules in a fluid of identical particles in thermal equilibrium in the limit of infinite dilution of the tagged particles. The diffusion equation describing this phenomenon on the macroscopic level can be derived ${ }^{(8,9)}$ from the kinetic equation (2.12) by constructing for given $\mathbf{q}$ the so-called hydrodynamic eigenfunction or diffusive mode of the operator $A-\mathbf{v}_{1} \cdot \partial / \partial \mathbf{r}_{1}$, i.e.,

$$
\Psi_{\mathbf{q}}^{\mathrm{D}}\left(x_{1}\right)=\left[\exp \left(-i \mathbf{q} \cdot \mathbf{r}_{1}\right)\right] \Psi^{\mathrm{D}}\left(\mathbf{q}, \mathbf{v}_{1}\right)
$$

satisfying $\lim _{q \rightarrow 0} \Psi^{\mathrm{D}}\left(\mathbf{q}, \mathbf{v}_{1}\right)=1$.

Treating $-\mathbf{v}_{1} \cdot \partial / \partial \mathbf{r}_{1}$ as a perturbation to $A$, one can expand both the eigenfunction $\Psi^{\mathrm{D}}$ and the corresponding eigenvalue $\omega^{\mathrm{D}}$ in powers of $i q$ as

$$
\begin{aligned}
\Psi^{\mathrm{D}}\left(\mathbf{q}, \mathbf{v}_{1}\right) & =\Psi_{0}^{\mathrm{D}}\left(\mathbf{v}_{1}\right)+i q \Psi_{1}^{\mathrm{D}}\left(\hat{\mathbf{q}}, \mathbf{v}_{1}\right)+(i q)^{2} \Psi_{2}^{\mathrm{D}}\left(\hat{\mathbf{q}}, \mathbf{v}_{1}\right)+\cdots \\
\omega^{\mathrm{D}} & =\omega_{0}^{\mathrm{D}}+i q \omega_{1}^{\mathrm{D}}+(i q)^{2} \omega_{2}^{\mathrm{D}}+\cdots
\end{aligned}
$$

with $\hat{\mathbf{q}}=\mathbf{q} /|\mathbf{q}|$. From standard perturbation theory one readily finds that $\omega_{0}^{\mathrm{D}}=\omega_{1}^{\mathrm{D}}=0$ and

$$
\omega_{2}^{\mathrm{D}} \equiv D=-\lim _{\varepsilon \rightarrow 0}\left\langle\varphi_{1}^{\mathrm{eq}}\left|v_{x} \frac{1}{A-\varepsilon} v_{x}\right| \varphi_{1}^{\mathrm{eq}}\right\rangle
$$

with

$$
\langle\varphi \mid \psi\rangle=\int d \mathbf{v}\left[\varphi_{1}^{\mathrm{eq}}(v)\right]^{-1} \varphi^{*}(\mathbf{v}) \psi(\mathbf{v})
$$

The explicit evaluation of (3.3) is rather involved and can be done either by means of the Chapman-Enskog expansion or, equivalently, by expanding $\Psi_{1}^{\mathrm{D}}\left(\varphi_{1}^{\mathrm{eq}}\right)^{-1}$ in a series of Sonine polynomials and determining the coefficients from a variational principle. The details of this procedure are 
described in the Appendix. The main result is that the coefficient of selfdiffusion assumes a form containing only equilibrium pair correlation functions at the values $r_{i j}=\sigma$ and $r_{i j}=R$ as unknown quantities. In principle, these pair correlation functions could be determined from equilibrium statistical mechanics. We decided, however, to use "experimental" values, i.e., values obtained from MD simulations done by one of the authors (J.M.). The expression for $D$ in lowest Enskog approximation is

$$
n^{*} D^{*}=\frac{3}{8} \pi^{-1 / 2} \frac{1}{g_{2}\left(\sigma^{+}\right)}\left[1+R^{* 2} \frac{g_{2}\left(R^{-}\right)}{g_{2}\left(\sigma^{+}\right)} \Xi(\beta \varepsilon)\right]^{-1}
$$

where $\Xi(x)=1-\frac{1}{2} x e^{-x}\left[1+e^{x / 2} K_{1}(x / 2)\right]$ and $K_{1}$ is a modified Bessel function ${ }^{(10)}$ and the reduced units $n^{*}, D^{*}$, and $R^{*}$ are defined at the beginning of the next section. The results for $g_{2}\left(\sigma^{+}\right)$are shown in Fig. 1. Extensive tables for both $g_{2}\left(\sigma^{+}\right)$and $g_{2}\left(R^{ \pm}\right)$are given in ref. 11 .

Corrections to (3.5) due to higher Enskog approximations are always small. The relative correction resulting from the second Enskog approximation is discussed in the Appendix and is shown in Fig. 2. It is seen to be largest in the absence of the square well, but even then it never exceeds a few percent. Still higher Enskog approximations yield even smaller corrections. Also notice that the inclusion of higher Enskog approximations always increases the value obtained for $D$ as a consequence of general principles of variational calculus. ${ }^{(9)}$

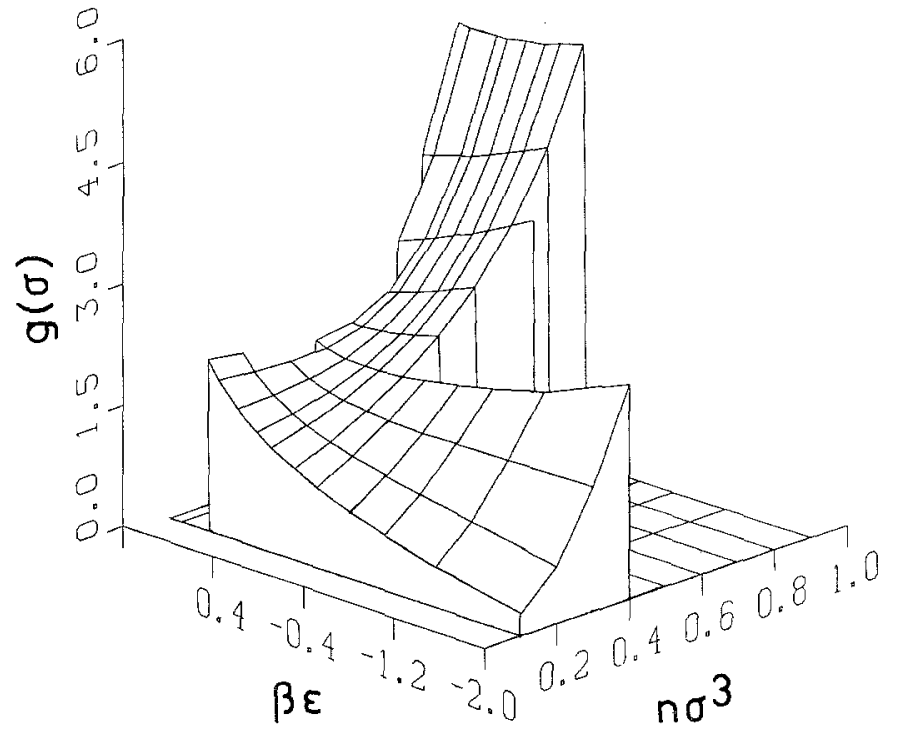

Fig. 1. The pair correlation function at the hard-core diameter $g_{2}\left(\sigma^{+}\right)$plotted as a function of $n^{*}$ and $\varepsilon^{*}$. 


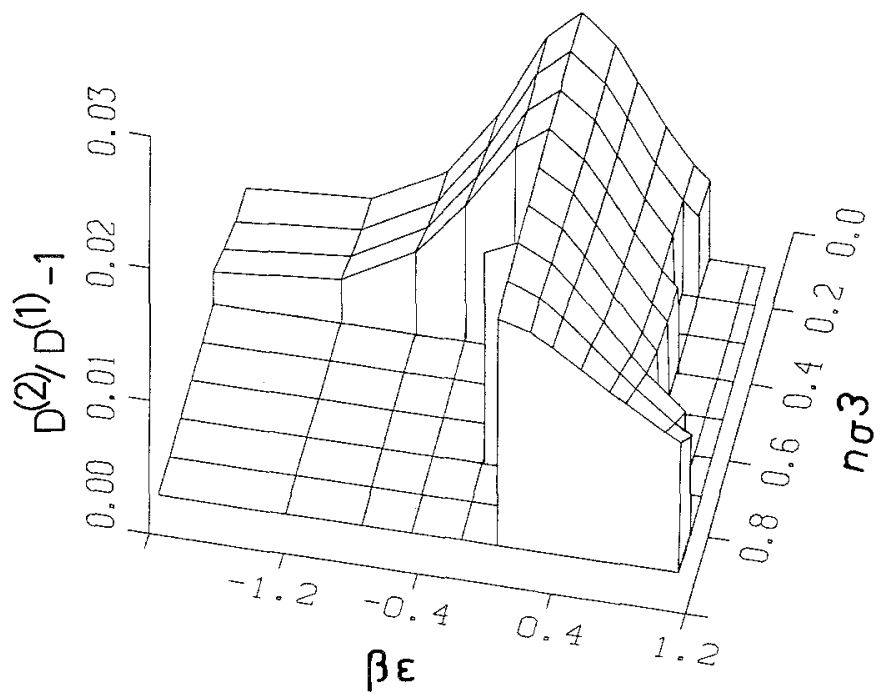

Fig. 2. The correction factor $D^{(2)} / D^{(1)}-1$, with $D^{(n)}$ the $n$th Enskog approximation to the diffusion coefficient, as a function of $n^{*}$ and $\varepsilon^{*}$.

\section{COMPARISON BETWEEN THEORY AND MOLECULAR DYNAMICS RESULTS}

The numerical results presented in this paper are expresed in natural reduced units, based on the hard-core diameter $\sigma$ as the unit of length and the unit of velocity $\left(k_{\mathrm{B}} T / m\right)^{1 / 2}$, which together determine the unit of time. This leads to the following reduced quantities: the outer-well diameter $R^{*}=R \sigma^{-1}$, the well depth $\varepsilon^{*}=\varepsilon\left(k_{\mathrm{B}} T\right)^{-1}=\beta \varepsilon$, the number density $n^{*}=n \sigma^{3}$, and the self-diffusion coefficient $D^{*}=D\left(\sigma^{2} k_{\mathrm{B}} T / m\right)^{-1 / 2}$.

For all figures presented here the outer well diameter is fixed at $R^{*}=1.5$. To eliminate trivial density dependences, it is useful to discuss $n^{*} D^{*}$ instead of $D^{*}$. The results of MD simulations for this quantity are plotted in Fig. 3 as a function of $n^{*}$ and $\varepsilon^{*}$. A part of these results has been discussed in a previous study, ${ }^{(12)}$ but new simulations (e.g., for the squareshoulder potential) have also been performed. The additional runs have been performed with the same method and over a comparable number of collisions. Therefore, the reader is referred to ref. 12 for a detailed description of the technique and for a discussion of its accuracy. Here we only mention that the most serious limitation of accuracy is due to the limited number of only 108 particles in the simulations. There are four important characteristics of Fig. 3 to be noted: 


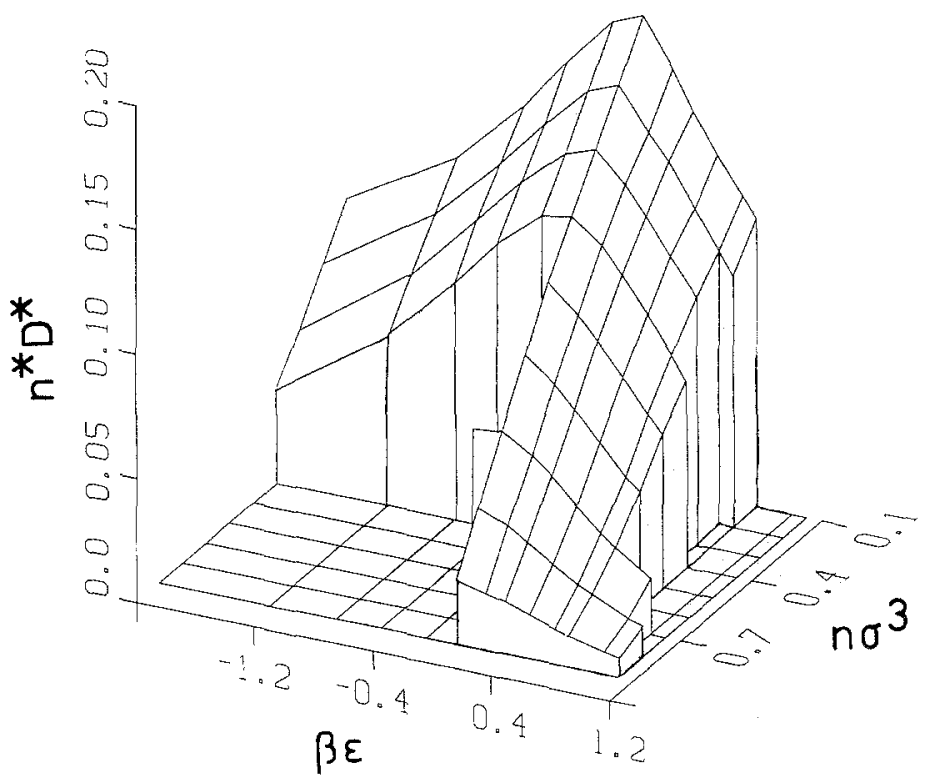

Fig. 3. Molecular dynamics results for $n^{*} D^{*}$ as a function of $n^{*}$ and $\varepsilon^{*}$.

1. At given density the maximum value of the functions is always reached at or very close to $\varepsilon=0$, that is, for hard-sphere conditions. The slope of $D^{*}$ as a function of $\varepsilon$ appears to jump at $\varepsilon=0$. This behavior is not counterintuitive: adding a square shoulder effectively increases the hardsphere diameter, which will impede diffusion, and adding a square well also increases the total cross section. In addition it makes particles stick together, which in general will also make diffusion harder. A discontinuity in slope may be caused by the possibility of having bound states in the square-well region, which stops for $\varepsilon=0$.

2. For square-shoulder values at constant density the slope decreases with increasing square-shoulder height. This behavior is easy to understand since, for $|\varepsilon| \gg k_{\mathrm{B}} T$, the system saturates as a hard-sphere system of radius $R^{*} \sigma$.

3. At constant temperature there is a nearly linear relationship between density and $n^{*} D^{*}$ similar to the Batschinski-Hildebrandt law ${ }^{(13,14)}$ for the relationship between reciprocal shear viscosity and density. ${ }^{(15)}$ In view of the near validity of Stokes law for molecular particles at liquid densities, ${ }^{(16)}$ this is not a surprising fact. Notice, however, that this type of relationship seems to hold for all values of $\varepsilon$ studied.

4. In the regime of higher densities and low temperatures the values 
of $n^{*} D^{*}$ as a function of $\varepsilon^{*}$ at constant densities have a point of inflection; for very low temperatures they approach zero.

In Fig. 4 the predictions of the kinetic theory for $n^{*} D^{*}$ are plotted against $\varepsilon^{*}$ and $n^{*}$. Regarding the same four characteristics discussed above, the following features catch the eye.

1. In contrast to the MD data at given density, the maximum is not always reached for hard-sphere values; at low densities the square-shoulder potential increases the diffusion constant compared to the hard-sphere values. We will comment on this later in this section.

2. Again there is a decreasing slope of the function values for constant densities in the square-shoulder regime, at least for $\varepsilon^{*}<\sim-0.5$.

3. Only for hard spheres does an approximately linear relation exist between density and $n^{*} D^{*}$; for $\varepsilon \neq 0$ there are strong deviations from this type of density dependence. Even for hard spheres this linear law is by far not as good as from MD data. This result is not surprising, since the Batchinski-Hildebrand law probably is the result of extended modecoupling effects, ${ }^{(17)}$ which are left out completely in the kinetic theory considered here.

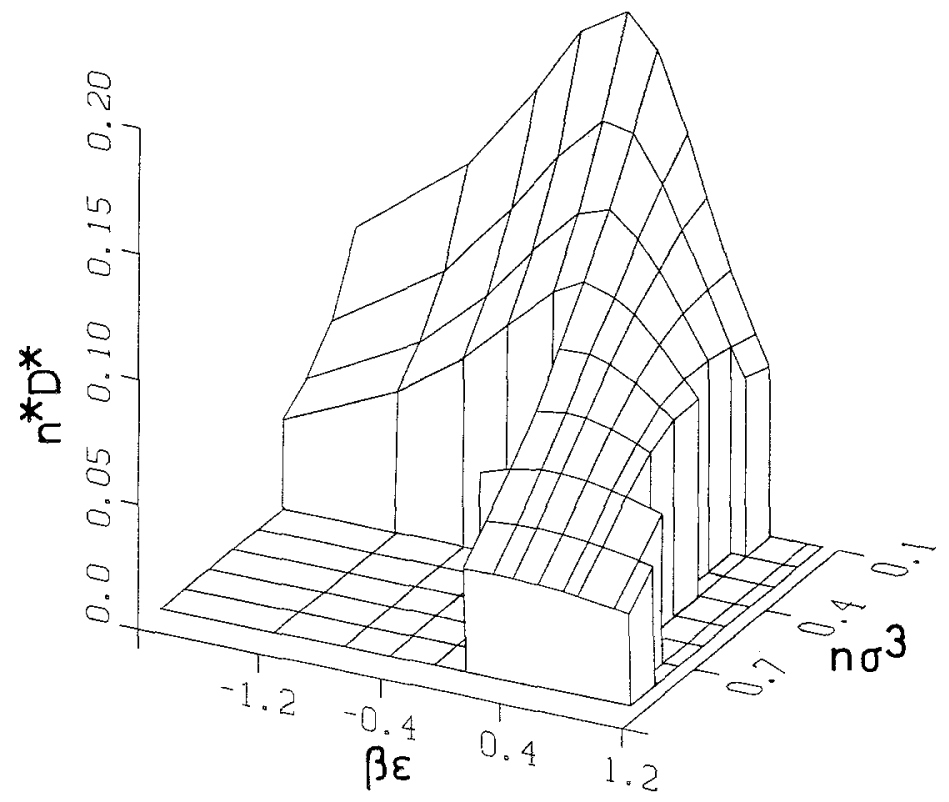

Fig. 4. Kinetic theory values for $n^{*} D^{*}$ as a function of $n^{*}$ and $\varepsilon^{*}$. 
4. There is a qualitative disagreement between theory and simulations in the regime of high densities and low temperatures. At constant density and variable temperature the diffusion coefficient is a concave function of $\varepsilon^{*}$ within the considered range of values. This difference could be due to the fact that the present kinetic theory does not consider the collective effects of clustering, except for their influence on the pair correlation function.

Figure 5 shows the ratio between the theoretical and the simulated diffusion coefficient. The failure of the kinetic theory at low temperatures and high densities is obvious: at high densities the theory predicts too high values for the diffusion coefficient. In contrast, at low densities the theoretical values are too low. Figure 6 shows a plot of four predictions for the dependence of $n^{*} D^{*}$ on $\varepsilon^{*}$ that may be helpful for understanding the low-density regime of the kinetic theory. The MD data at density $n^{*}=0.1$ (triangles) are compared with kinetic theory evaluated using the MD values for the pair correlation function at density $n^{*}=0.1$ (pluses) and with the kinetic theory using $g_{2}\left(\sigma^{+}\right)$, respectively $g_{2}\left(R^{-}\right)=e^{\beta \varepsilon}$ (squares), as input. The latter case corresponds to the limit $n^{*} \rightarrow 0$. For comparison the Boltzmann-theory predictions are plotted (octagons). ${ }^{(18)}$ The main difference between Boltzmann theory and our kinetic theory is that in our theory all collisions at discontinuities of the potential are uncorrelated, but in the Boltzmann case only sequences of collisions that describe a complete scattering process are uncorrelated; during the scattering process,

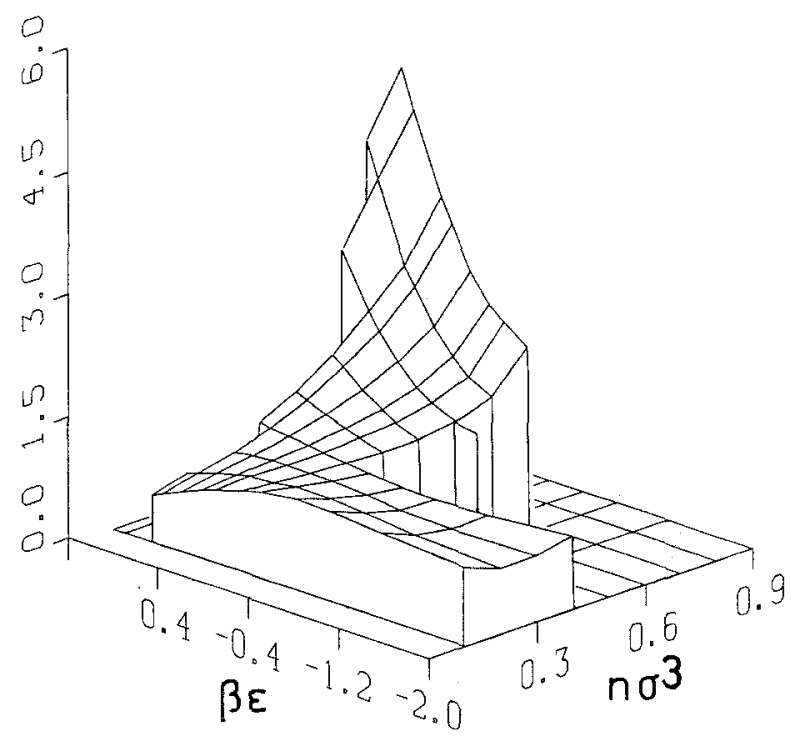

Fig. 5. Ratio between kinetic theory and MD values of $n^{*} D^{*}$ as a function of $n^{*}$ and $\varepsilon^{*}$. 


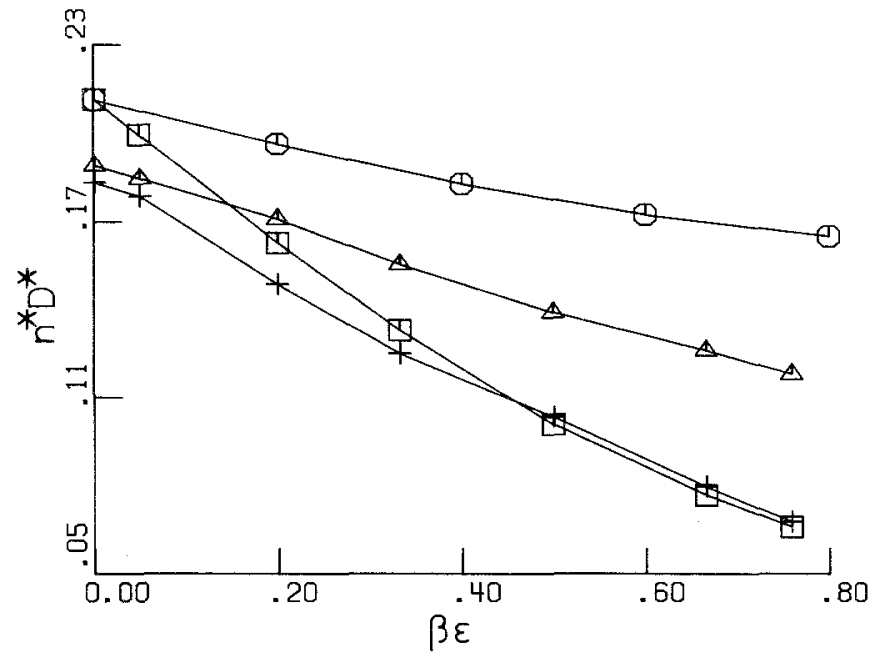

Fig. 6. The dependence of $n^{*} D^{*}$ on $\varepsilon^{*}$ at $n^{*}=0.1$, as obtained from $(\triangle)$ MD simulations, $(O)$ the Boltzmann equation, $(+)$ our kinetic theory with $g_{2}\left(\sigma^{+}\right)$and $g_{2}\left(R^{-}\right)$obtained from MD simulations, and $(\square)$ our kinetic theory with low-density values for $g_{2}\left(\sigma^{+}\right)$and $g_{2}\left(R^{-}\right)$.

correlations among entering, reflecting at the hard core, and leaving the square well are not ignored. Of course, one hopes that in the regime of higher densities collisions with third particles will indeed destroy all correlations still present in the Boltzmann equation. Since no predictions of transport properties for square-shoulder molecules based on the Boltzmann kinetic theory are available, only the interval of positive values of $\varepsilon$ was plotted. The predictions of our kinetic theory in the limit $n^{*} \rightarrow 0$ are proportional to $e^{\varepsilon^{*}}$ at $\varepsilon^{*}=0$; the nonzero slope for $n^{*}=0.1$ is entirely due to the $\varepsilon$ dependence of the pair correlation functions. Obviously, the deviations between theory and MD results at low density are largely due to the neglect of correlations between subsequent partial collisions of identical pairs of particles. ${ }^{(19)}$

For high temperatures an expansion in $\varepsilon$ of the expressions for $n^{*} D^{*}$ for both our kinetic theory and the Boltzmann results may be helpful. For our kinetic theory an expansion of the function $\Xi\left(\varepsilon^{*}\right)$ setting $g_{2}\left(R^{-}\right) / g_{2}\left(\sigma^{+}\right)=1$ yields

$$
\Xi\left(\varepsilon^{*}\right)=\varepsilon^{* 2}\left(\frac{7}{16}-\frac{1}{8} \log \left(\frac{\varepsilon^{*}}{4}\right)-\frac{1}{8} \gamma\right)+\cdots
$$

where $\gamma$ is Euler's constant. The expansion starts with an almost quadratic term in $\varepsilon$. The predictions of the Boltzmann theory for $R^{*}=1.5 \mathrm{are}^{(20)}$

$$
n^{*} D^{*}=\frac{3}{8} \pi^{-1 / 2}\left[1+\widetilde{\Xi}\left(\varepsilon^{*}\right)\right]^{-1}
$$


with

$$
\begin{aligned}
\tilde{\Xi}\left(\varepsilon^{*}\right) & =\varepsilon^{*} f_{1}+\varepsilon^{* 2} \ln \left(\varepsilon^{*}\right) f_{2}+\varepsilon^{* 2} f_{3} \\
f_{1} & =-\frac{7}{4}+\frac{155}{128} \ln (5) \approx 0.18 \\
f_{2} & =-\frac{9}{16} \\
f_{3} & =-\frac{17}{64}+\frac{9}{16}[\ln (4)-\gamma]+\frac{45}{32} \ln \left(\frac{18}{5}\right)-\frac{259}{256} \ln (5)
\end{aligned}
$$

Although, as stated above, the Boltzmann equation for the squareshoulder potential has not been solved explicitly, one easily convinces oneself that on expanding $D^{*}$ as a function of $\varepsilon^{*}$ the linear term is the same as for the square-well potential. Hence $D^{*}$ in the limit $n^{*} \rightarrow 0$ has a maximum on the square-shoulder side. Our MD simulations do not capture this, because the lowest density considered still shows appreciable deviations from the Boltzmann results. The theory, on the other hand, exaggerates this effect and yields the maximum for $D^{*}$ at $\varepsilon^{*}=0$ for densities larger than $n^{*}=0.2$ only.

On the square-well side at large $\varepsilon^{*}$ our theory severely underestimates $D^{*}$, which must be an effect of the existence of bound states. This may at first seem surprising, since one would expect the ignorance of bound states to result in an overestimate of $D^{*}$. The following explanation seems plausible, however. As one sees from (3.5), our theory yields $D^{*}$ inversely proportional to $g_{2}\left(\sigma^{+}\right)$, or, equivalently, to the collision frequency. In reality, collisions between bound pairs have little effect on the diffusion process, since they do not affect the center-of-mass velocity of the pair. The MD simulations do not distinguish collisions between bound and unbound pairs of particles; bound particles clearly have a higher than average collision rate, hence the collision rate of the unbound particles is estimated too high. Indeed, the theory also does not take into account the reduction in diffusion rate as a consequence of being in a bound state, but apparently the overestimate of the collision frequency of unbound particles is the dominant effect.

\section{THE VELOCITY AUTOCORRELATION FUNCTION}

The normalized velocity autocorrelation function (VACF) defined as

$$
\Gamma(t):=\left\langle v_{1_{x}}(0) v_{1_{x}}(t)\right\rangle_{\mathrm{eq}} /\left\langle\left[v_{1_{x}}(0)\right]^{2}\right\rangle_{\text {eq }}
$$


contains much more information on the dynamics of our system than the transport coefficients alone. The VACF is related to the coefficient of selfdiffusion through the Green-Kubo formula:

$$
D=\frac{1}{m \beta} \int_{0}^{\infty} d t \Gamma(t)
$$

The function $\Gamma(t)$ satisfies the following three exact relations:

$$
\begin{aligned}
\Gamma(0) & =1 \\
\left.\frac{\partial}{\partial t} \Gamma(t)\right|_{t=0} & =\beta m\left\langle\varphi_{1}^{\mathrm{eq}}\left|v_{x} A v_{x}\right| \varphi_{1}^{\mathrm{eq}}\right\rangle \\
\lim _{t \rightarrow \infty} \Gamma(t) & =0
\end{aligned}
$$

The first relation is a trivial consequence of the definition of the VACF; the second formula states that matrix elements of our kinetic operator determine the slope of the VACF at the origin exactly. The fact that this slope is not zero is a peculiar consequence of the discontinuities in our pair interaction potential, giving rise to instantaneous jumps in the particle velocities. Table I shows a test of Eq. (5.3b). The initial slope of the VACF obtained from our MD simulations was determined by a least squares fit using a smoothed cubic splines method and compared to our theoretical expression. One sees that the MD results are correct within their statistical precision.

Table I. Initial Slope of the VACF As Determined from MD Simulations $\left[\Gamma^{\prime}(0)\right]$ versus Its Theoretical Value $\left[-1 / D^{*(1)}\right]$

Evaluated with the Use of MD Pair Correlation Functions

\begin{tabular}{clll}
\hline$n^{*}$ & $\varepsilon^{*}$ & $\Gamma^{\prime}(0)$ & $-1 / D^{*(1)}$ \\
\hline 0.1 & -2.0 & -1.1245 & -1.1278 \\
& -1.25 & -0.8172 & -0.8122 \\
& -0.8 & -0.6344 & -0.6302 \\
& -0.5 & -0.5404 & -0.5383 \\
& -0.2 & -0.4906 & -0.5066 \\
& -0.05 & -0.5291 & -0.5276 \\
0.0 & -0.5480 & -0.5419 \\
0.05 & -0.5575 & -0.5625 \\
0.2 & -0.6491 & -0.6529 \\
& 0.333 & -0.7825 & -0.7721 \\
& 0.5 & -0.9735 & -0.9730 \\
0.667 & -1.2748 & -1.2572 \\
& 0.76 & -1.4810 & -1.4775 \\
\hline
\end{tabular}


Table 1. (Continued)

\begin{tabular}{|c|c|c|c|}
\hline$n^{*}$ & $\varepsilon^{*}$ & $\Gamma^{\prime}(0)$ & $-1 / D^{*(1)}$ \\
\hline 0.2 & $\begin{array}{l}-2.0 \\
-1.25 \\
-0.8 \\
-0.5 \\
0.0 \\
0.2 \\
0.333 \\
0.5 \\
0.667 \\
0.760\end{array}$ & $\begin{array}{l}-3.3776 \\
-2.2431 \\
-1.6805 \\
-1.4054 \\
-1.2713 \\
-1.4346 \\
-1.6143 \\
-1.9235 \\
-2.4294 \\
-2.8145\end{array}$ & $\begin{array}{l}-3.3690 \\
-2.2620 \\
-1.6779 \\
-1.3931 \\
-1.2540 \\
-1.4166 \\
-1.6057 \\
-1.9421 \\
--2.4286 \\
-2.7994\end{array}$ \\
\hline 0.3 & $\begin{array}{l}-2.0 \\
-1.25 \\
-0.8 \\
-0.5 \\
-0.2 \\
0.0\end{array}$ & $\begin{array}{r}-6.7722 \\
-4.3926 \\
-3.2450 \\
2.6307 \\
-2.2618 \\
-2.2006\end{array}$ & $\begin{array}{l}-6.7656 \\
-4.4144 \\
-3.2510 \\
-2.6339 \\
-2.2628 \\
-2.1933\end{array}$ \\
\hline 0.4 & 0.0 & -3.4990 & -3.4510 \\
\hline 0.5 & $\begin{array}{l}0.0 \\
0.2 \\
0.333 \\
0.5 \\
0.667 \\
0.76\end{array}$ & $\begin{array}{l}-5.2101 \\
-5.2185 \\
-5.2359 \\
-5.5550 \\
-6.1510 \\
-6.3665\end{array}$ & $\begin{array}{l}-5.1490 \\
-5.1167 \\
-5.2498 \\
-5.5561 \\
-6.0370 \\
-6.3878\end{array}$ \\
\hline 0.6 & $\begin{array}{l}0.0 \\
0.2 \\
0.333 \\
0.5 \\
0.667 \\
0.76 \\
0.9\end{array}$ & $\begin{array}{l}-7.4943 \\
-7.3174 \\
-7.3768 \\
-7.5069 \\
-7.9478 \\
-8.2441 \\
-8.7200\end{array}$ & $\begin{array}{l}-7.4798 \\
-7.2970 \\
-7.3393 \\
-7.5396 \\
-7.9316 \\
-8.1889 \\
-8.6668\end{array}$ \\
\hline 0.7 & $\begin{array}{l}0.0 \\
0.2 \\
0.333 \\
0.5 \\
0.667 \\
0.76 \\
0.9 \\
1.0\end{array}$ & $\begin{array}{l}-10.756 \\
-10.462 \\
-10.315 \\
-10.502 \\
-10.828 \\
-10.962 \\
-11.422 \\
-11.644\end{array}$ & $\begin{array}{l}-10.720 \\
-10.417 \\
-10.383 \\
-10.523 \\
-10.783 \\
-10.999 \\
-11.395 \\
-11.718\end{array}$ \\
\hline 0.8 & 0.0 & -15.338 & -15.324 \\
\hline 0.86 & 0.0 & -19.001 & - \\
\hline 0.9 & 0.0 & -21.890 & -21.901 \\
\hline 1.1 & 0.0 & -27.837 & - \\
\hline
\end{tabular}


The third relation $(5.3 \mathrm{c})$ must be due to the fact that a system with hard-core repulsions is a mixing system. ${ }^{(21)}$ To lowest order (first Enskog approximation), our kinetic theory predicts an exponentially decreasing $\mathrm{VACF}$

$$
\Gamma(t)=e^{-x t}
$$

with $\alpha^{-1} \approx D^{*}$ if $t$ is expressed in the dimensionless units introduced in Section 4 .

In Figs 7 and 8 the VACF is plotted for several temperatures at the densities $n^{*}=0.1$ and $=0.7$. The MD-VACF shows at least four qualitative deviations from a simple exponential decay.

\subsection{Bound States}

At low densities and temperatures one sees the influence of bound states. Figure 9 shows the logarithm of the time derivative of the VACF at $n^{*}=0.1$. The effects of bound states are demonstrated by its oscillatory behavior. At densities higher than $n^{*}=0.2$ no bound state effects can be recognized.

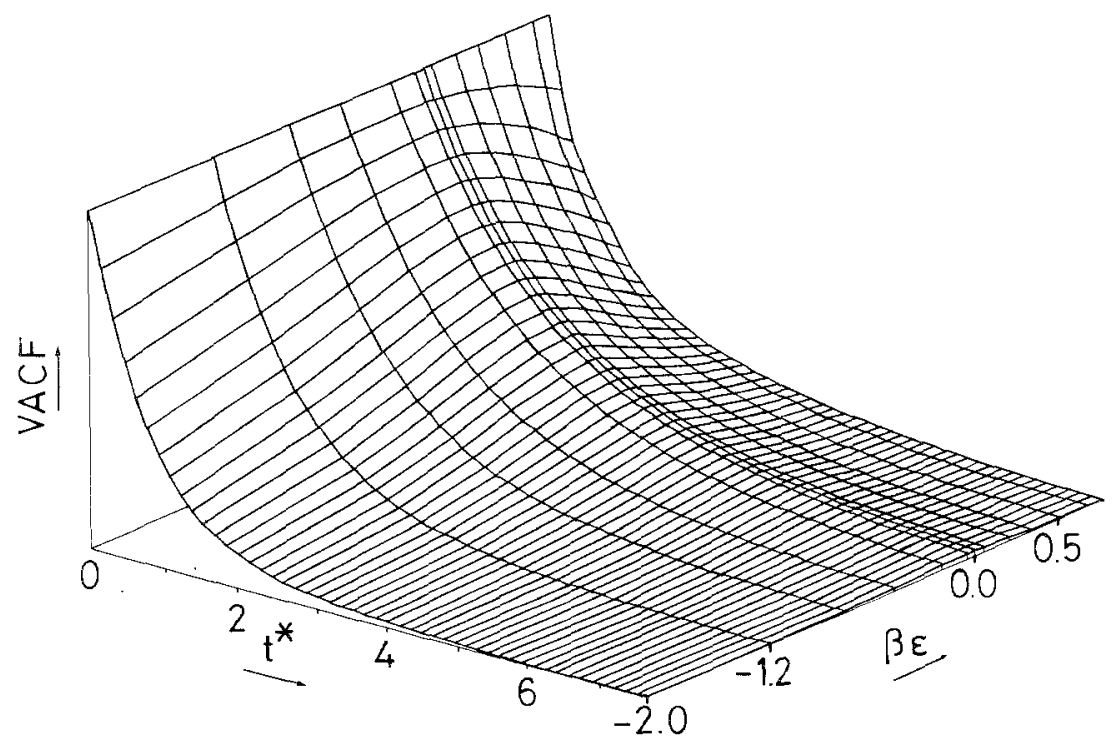

Fig. 7. The velocity autocorrelation function as a function of reduced time $t^{*}$ for several values of $\varepsilon^{*}$ at $n^{*}=0.1$. 


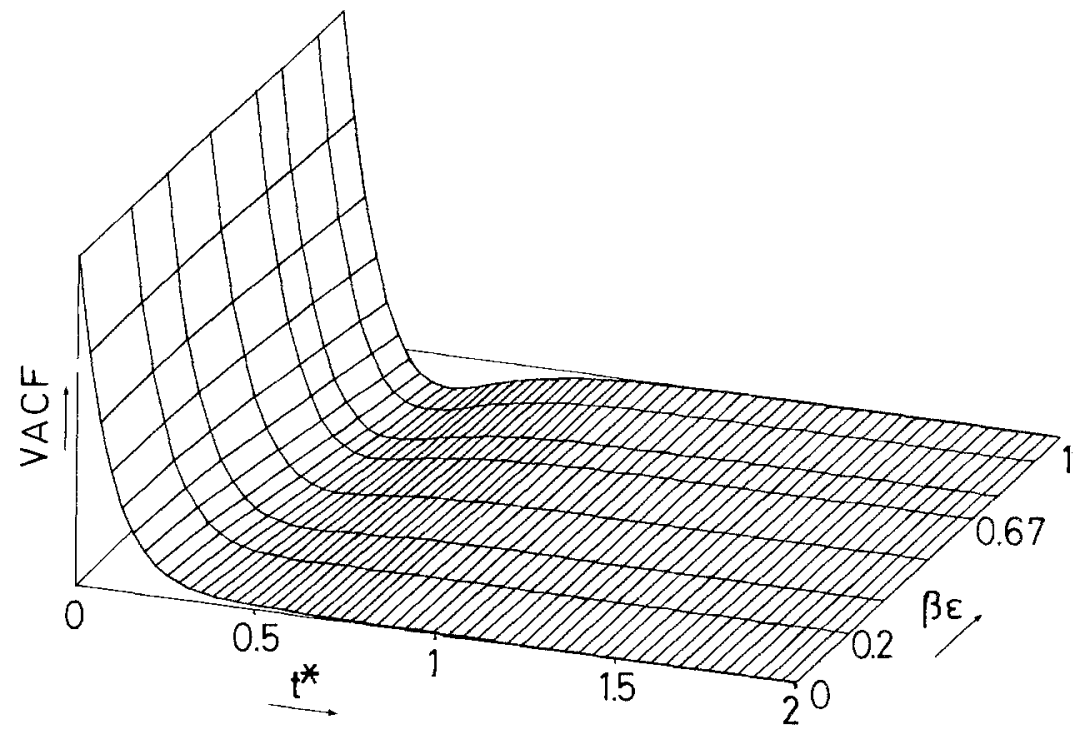

Fig. 8. As in Fig. 7, at the density $n^{*}=0.7$

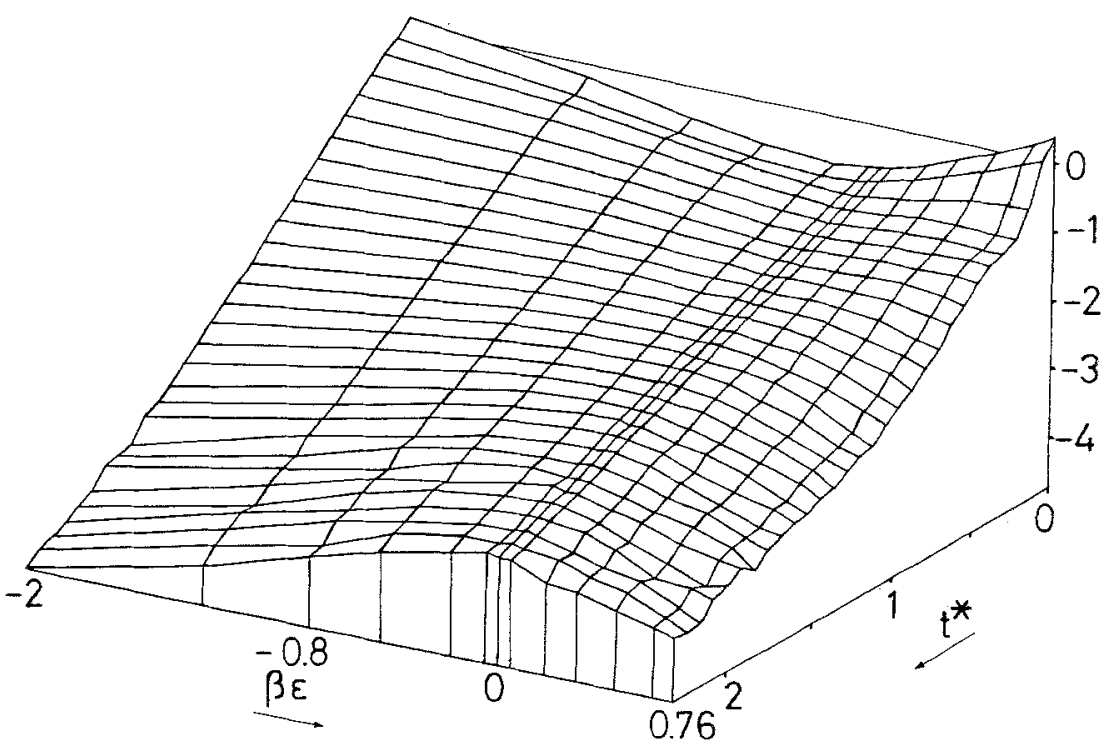

Fig. 9. The logarithm of the time derivative of the VACF as a function of $t^{*}$ at $n^{*}=0.1$. Bound-state effects manifest themselves through oscillatory behavior. 


\subsection{Another Slowly Decaying Exponential Mode}

Figures 10 and 11 show a logarithmic plot of the VACF at several temperatures and at the densities $n^{*}=0.1$ and $=0.6$. In the high-temperature (low- $\varepsilon$ ) regime at $n^{*}=0.6$ a second interval in which the logarithm of the VACF can be approximated by a linear function is clearly seen. It is important to notice that this second exponential decay is much slower than the first one. Therefore, higher Enskog approximations, which result in new, faster-decreasing exponentials, cannot explain the phenomenon.

\subsection{The Negative Minimum}

Figure 8 shows that there is a negative minimum in the VACF at high densities and low temperatures. One possible explanation for the negative minimum is the cage effect: A particle that moves straight on in a dense fluid generates a density fluctuation. There is a greater probability to find no particle just behind the moving particle than in front of it. Therefore, after some time there is a higher probability to be moving opposite to the original direction within the "vacuum" left behind than to continue in the original direction. In this connection also notice that for motion in a

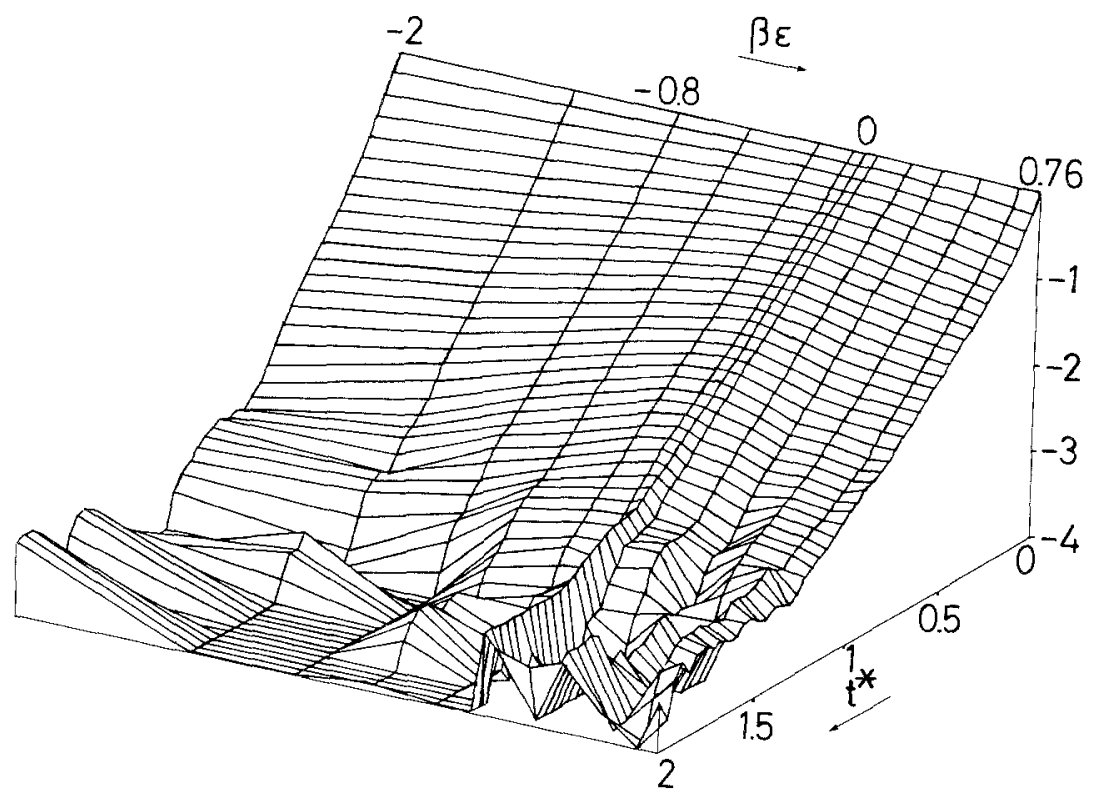

Fig. 10. The logarithm of the VACF as a function of $t^{*}$ at $n^{*}=0.1$. 


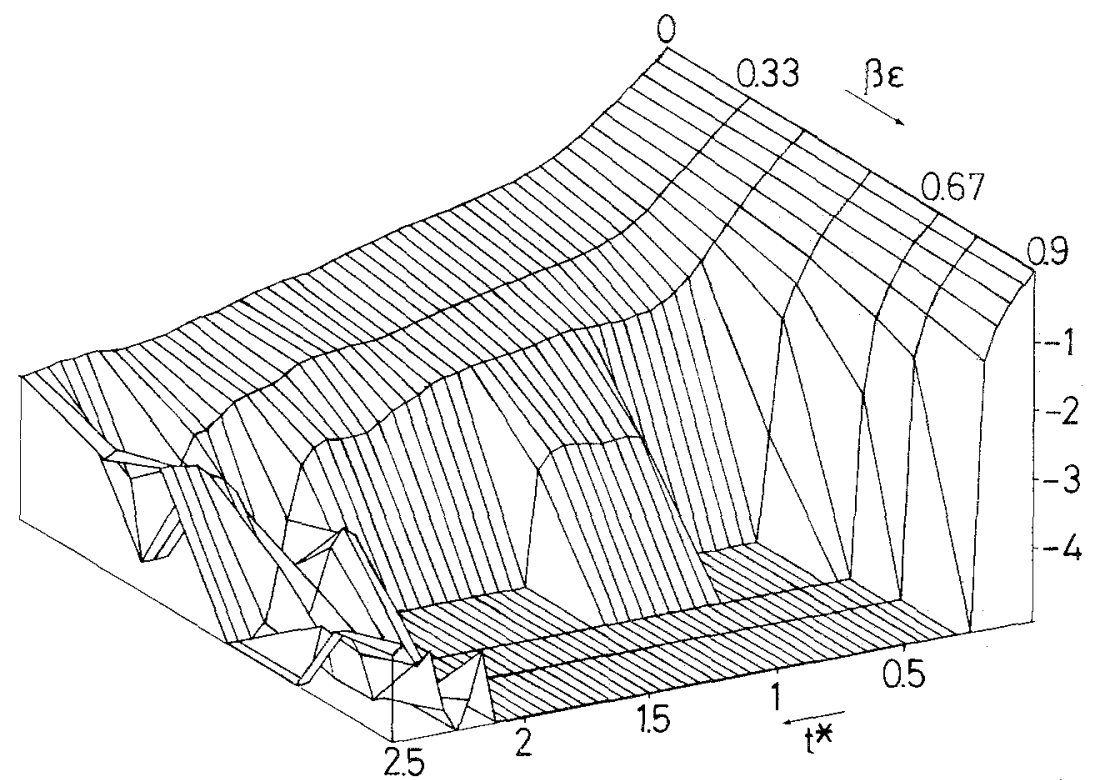

Fig. 11. As in Fig. 10, at $n^{*}=0.6$. A second regime of exponential decay is seen for not too large $\varepsilon^{*}$ and $t^{*}$ between 0.5 and 2 .

perfect cage the time integral of the VACF has to be zero because of (5.2), since the diffusion coefficient vanishes. Hence the VACF, which always starts out positive, has to become negative at larger times. A more quantitative explanation of the negative minimum can be derived from extended mode coupling theory. ${ }^{(22)}$ This theory attributes the minimum to a coupling of the tagged particle velocity to products of a diffusive mode for the tagged particle motion and an extended heat mode for the motion of the fluid. It should be noted that a negative minimum already appears in the case of a hard-sphere fluid at densities of $n^{*}=0.8-0.9$, but the effect is much more pronounced in the presence of an attractive potential. It is seen already at lower densities and at equal densities the minimum is much deeper for the square-well system than for the pure hard-sphere system.

\subsection{Long-Time Tails}

MD simulations at hard-sphere systems with many particles $(\sim 4000)$ demonstrate the presence of algebraically decaying long-time tails. ${ }^{(23)}$ The limited number of particles in our simulation prevents us from observing this phenomenon in our results, but since the long-time tails can be predic- 
ted quite generally from hydrodynamic arguments, they should be present in the square-well system as well.

We conclude that the behavior of the VACF for the square-well fluid not only is much more complicated than the almost exponential decay resulting from the Boltzmann equation, but even shows much more complexity than pure hard-sphere systems at comparable densities. At low densities this is mainly due to the occurrence of bound states, whereas at high densities mode coupling and extended mode coupling effects appear to be important. The latter seem to give rise to much more pronounced negative minima in the VACF than those found for pure hard-sphere systems. In addition, we found the appearance of a second exponential regime of slower decay rate at reasonably high densities. Our speculation is that these are due to collective effects involving moderate numbers of particles, e.g., the motion of particle complexes, but we have no further evidence to support this.

\section{DISCUSSION}

Our comparison between an Enskog-type kinetic theory for a squarewell fluid and the results of molecular dynamics simulations reveals that the region of applicability of the kinetic theory is rather restricted. Around $n^{*}=0.3$ the theory works reasonably well; the relative change of $D^{*}$ as a function of $\varepsilon^{*}$ is described fairly accurately, although an underestimation of $D^{*}$ by about $10 \%$, already present in the hard-sphere case, persists throughout the square-well regime. At lower densities our theory underestimates $D^{*}$ as a result of the overestimation of the collision frequency for unbound particles we discussed in Section 4. For higher densities our theory increasingly overestimates $D^{*}$ with growing $\varepsilon^{*}$. This obviously must be due to the neglect of extended mode coupling contributions, which, as seen in the previous section, produce a negative minimum in the VACF of a magnitude that increases rapidly with $\varepsilon^{*}$.

A good theoretical description of the square-well system at high densities may be obtainable by adding ring terms and perhaps repeated ring terms to the Enskog-like kinetic equation discussed here, since these terms do account for mode coupling and extended mode coupling effects. ${ }^{(22)}$ Similarly, at moderate densities, i.e., $n^{*}<0.3$, a theory is needed that accounts for correlations between partial collisions among the same pair of particles and especially takes bound states into consideration. ${ }^{(24)}$ In either case the theory treated here will be an essential ingredient of a more complicated theoretical framework, and in addition will serve as an important reference point in limiting cases.

Finally, notice that for the square-shoulder potential in all cases 
investigated here the agreement between theory and simulation results is markedly better than for the square-well potential. It is roughly of the same quality as the agreement between Enskog's theory and the simulation results for hard spheres. The following feature is remarkable, however: as $\varepsilon^{*}$ runs between 0 and $-\infty$, the square-shoulder system interpolates between two hard-sphere systems at different dimensionless densities. Yet at intermediate values of $\varepsilon^{*}$ (notably $\varepsilon^{*}=2$ at $n^{*}=0.2$ and $\varepsilon^{*}=1.25$ at $n^{*}=0.3$ ) theory underestimates $D^{*}$ by more than ever happens for a pure hard-sphere system at densities intermediate between the limiting ones.

Obviously in the treatment of realistic fluids the interparticle attractions offer some of the hardest problems one has to deal with and on the kinetic theory level quite complicated equations seem needed to do so.

\section{APPENDIX}

In this Appendix we approximately evaluate the formal expression for the coefficient of self-diffusion

$$
D=-\lim _{v \rightarrow 0}\left\langle\varphi^{\mathrm{eq}}(v) v_{x}\left|\frac{1}{A-v}\right| v_{x} \varphi^{\mathrm{eq}}(v)\right\rangle
$$

It may be considered as a matrix element of $(\Lambda-v)^{-1}$ under the inner product (3.4). One way to calculate this matrix element approximately is by replacing $A$ by a finite-dimensional matrix $A_{n}$ defined on a suitably chosen basis $\varphi_{i}(i=1, \ldots, n)$ and inverting this matrix. The most common choice for the function $\varphi_{i}$ is

$$
\varphi_{i}(\mathbf{v})=\left[\frac{\Gamma(i) \Gamma(5 / 2) m}{\Gamma(i+3 / 2) k_{\mathrm{B}} T}\right]^{1 / 2} v_{x} S_{3 / 2}^{(i-1)}\left(\frac{m v^{2}}{2 k_{\mathrm{B}} T}\right) \varphi^{\mathrm{eq}}(v)
$$

where $S_{3 / 2}^{(r)}$ is a Sonine polynomial. ${ }^{(9)}$ In the solution of the Boltzmann equation this choice inserted into $A_{n}$ yields the so-called $n$th Enskog approximation to the diffusion coefficient. ${ }^{(9,25)}$ The functions $\varphi_{i}$ are orthonormal with respect to the inner product (3.4), that is,

$$
\left\langle\varphi_{i}(\mathbf{v}) \mid \varphi_{j}(\mathbf{v})\right\rangle=\delta_{i j}
$$

As $\varphi_{1}(\mathbf{v})=\left(m / k_{\mathrm{B}} T\right)^{1 / 2} v_{x} \varphi^{\mathrm{eq}}(v)$, we have

$$
D=-k_{\mathrm{B}} T / m \lim _{v \rightarrow 0}\left\{(\Lambda-v)^{-1}\right\}_{11}
$$

Denoting the matrix elements of $A$ with respect to $\varphi_{i}$ as $A_{i j}$, we find the following approximate expressions for $D$ : 
For $n=1$

$$
D^{(1)}=-\frac{k_{\mathrm{B}} T}{m \Lambda_{11}}
$$

For $n=2$

$$
D^{(2)}=-\frac{k_{\mathrm{B}} T}{m \Lambda_{11}}\left(1+\frac{\Lambda_{12}^{2}}{\Lambda_{11} \Lambda_{22}-\Lambda_{12}^{2}}\right)
$$

etc.

These are well-known results for the first and second Enskog approximation to $D .^{(9)}$

The calculation of the matrix elements $A_{i j}$ remains to be done. With the aid of (2.12) these can be written as

$$
A_{i j}=\int d \mathbf{v}_{1} \int d \mathbf{v}_{2} \int d \mathbf{r}_{2}\left\{\varphi^{\mathrm{eq}}\left(v_{1}\right)\right\}^{-1} \varphi_{i}\left(\mathbf{v}_{1}\right) \bar{T}_{12} g_{2}\left(r_{12}\right) n \varphi^{\mathrm{eq}}\left(v_{2}\right) \varphi_{j}\left(\mathbf{v}_{1}\right)
$$

After a lengthy calculation, which was largely performed on a computer, we found for the square-well potential

$$
A_{i j}=-\frac{1}{t_{0}}\left[g_{2}\left(\sigma^{+}\right) \Omega_{i j}(\infty)+R^{* 2} g_{2}\left(R^{-}\right) \Omega_{i j}\left(\varepsilon^{*}\right)\right]
$$

with

$$
t_{0}=\left(\frac{m}{\pi k_{\mathrm{B}} T}\right)^{1 / 2} \frac{1}{4 n \sigma^{2}}
$$

and

$$
\begin{aligned}
\Omega_{11}\left(\varepsilon^{*}\right)= & \frac{2}{3}\left[1-\frac{\varepsilon^{*}}{2} e^{-\varepsilon^{* / 2}} K_{1}\left(\frac{\varepsilon^{*}}{2}\right)-\frac{\varepsilon^{*}}{2} e^{-\varepsilon^{*}}\right]=\frac{2}{3} \Xi\left(\varepsilon^{*}\right) \\
\Omega_{12}\left(\varepsilon^{*}\right)= & \frac{-1}{3 \sqrt{10}}\left\{\Xi\left(\varepsilon^{*}\right)-\varepsilon^{* 2}\left[\frac{e^{-\varepsilon^{*} / 2}}{2} K_{0}\left(\frac{\varepsilon^{*}}{2}\right)+e^{-\varepsilon^{*}}\right]\right\} \\
\Omega_{22}\left(\varepsilon^{*}\right)= & \frac{59}{60} \Xi\left(\varepsilon^{*}\right)+e^{-\varepsilon^{* / 2}}\left(\frac{13}{60}-\frac{1}{30} \varepsilon^{* 3}\right) \\
\Omega_{13}\left(\varepsilon^{*}\right)= & \frac{-1}{12 \sqrt{70}}\left\{\Xi\left(\varepsilon^{*}\right)-\varepsilon^{* 2} e^{-\varepsilon^{*} / 2}\left[2 K_{0}\left(\frac{\varepsilon^{*}}{2}\right)-\varepsilon^{*} K_{1}\left(\frac{\varepsilon^{*}}{2}\right)\right]\right. \\
& \left.-e^{-\varepsilon^{*}}\left(4 \varepsilon^{*}-2 \varepsilon^{* 3}\right)\right\}
\end{aligned}
$$




$$
\begin{aligned}
\Omega_{23}\left(\varepsilon^{*}\right)= & \frac{-1}{\sqrt{63}}\left\{\frac{139}{80} \Xi\left(\varepsilon^{*}\right)-\frac{\varepsilon^{* 2}}{2} e^{-\varepsilon^{* / 2}} K_{0}\left(\frac{\varepsilon^{*}}{2}\right)\right. \\
& \left.-e^{-\varepsilon^{*}}\left(\frac{177}{80} \varepsilon^{*^{2}}-\frac{3}{5} \varepsilon^{* 3}+\frac{1}{20} \varepsilon^{* 4}\right)\right\} \\
\Omega_{33}= & \frac{559}{448} \Xi\left(\varepsilon^{*}\right)-e^{-\varepsilon^{*}}\left(-\frac{213}{560} \varepsilon^{*^{2}}+\frac{247}{1680} \varepsilon^{* 3}-\frac{13}{560} \varepsilon^{* 4}+\frac{1}{840} \varepsilon^{* 5}\right)
\end{aligned}
$$

For the square-shoulder potential one has to replace $g_{2}\left(R^{-}\right)$by $g_{2}\left(R^{+}\right)$; otherwise the expressions remain the same.

\section{ACKNOWLEDGMENTS}

We thank K. W. Kehr, J. Anlauf, and G. Kneller for assistance in using the computer algebra system of the Zentralinstitut für angewandte Mathematik of the KFA in Jülich. We acknowledge support by NATO Research Grant No. 419/82.

\section{REFERENCES}

1. B. J. Alder and T. E. Wainwright, Phys. Rev. Lett. 18:988 (1967).

2. D. Enskog, K. Sven. Vetenskapsakad. Handl. 63:4 (1922).

3. H. van Beijeren and M. H. Ernst, Physica 68:437 (1973); 70:225 (1973).

4. W. E. Alley and B. J. Alder, Phys. Rev. A 27:3158 (1983); W. E. Alley, B. J. Alder, and S. Yip, Phys. Rev. A 27:3174 (1983); E. G. D. Cohen, B. Kamgar-Parsi, and I. M. de Schepper, Phys. Lett. 114A:241 (1986).

5. I. M. de Schepper, J. C. van Rijs, A. H. van Well, P. Verkerk, L. A. de Graaf, and C. Bruin, Phys. Rev. A 29:1602 (1984).

6. H. T. Davis, S. A. Rice, and J. V. Sengers, J. Chem. Phys. 35:2210 (1961).

7. J. Karkheck, H. van Beijeren, I. M. de Schepper, and G. Stell, Phys. Rev. A 32:2517 (1985).

8. E. H. Hauge, in Transport Phenomena, G. Kirczenow and J. Marro, eds. (Springer, Berlin), p. 337.

9. P. Résibois and M. de Leener, Classical Kinetic Theory of Fluids (Wiley, New York, 1977).

10. I. S. Gradshteyn and I. M. Ryzhik, Table of Integrals, Series and Products (Academic Press. New York, 1965).

11. H. Wilbertz, Diplomarbeit, RWTH Aachen (unpublished).

12. J. P. J. Michels and N. J. Trappeniers, Physica 116A:316 (1982).

13. A. J. Batschinsky, Z. Phys. Chem. 84:643 (1913); J. H. Hildebrand, Viscosity and Diffusivity (Wiley, New York, 1976).

14. J. J. van Loef, Physica 124B:305 (1984); P. S. van der Gulik and N. J. Trappeniers, Physica 135A:1 (1986).

15. J. P. J. Michels and N. J. Trappeniers, Physica 90A:179 (1978).

16. E. McLaughlin, Trans. Faraday Soc. 55:28 (1959); R. Zwanzig and M. Bixon, Phys. Rev. A 2:2005 (1970).

17. I. M. de Schepper, A. F. E. M. Haffmans, and H. van Beijeren, Phys. Rev. Lett. 57:1715 (1986). 
18. J. O. Hirschfelder, C. F. Curtiss, and R. B. Bird, Molecular Theory of Gases and Liquids (Wiley, New York, 1967).

19. M. C. Marchetti and J. W. Dufty, Chem. Phys. Lett. 70:539 (1980).

20. J. Karkheck and G. Stell, J. Phys. Chem. 87:2858 (1983).

21. Ya. G. Sinai, Introduction to Ergodic Theory (Princeton University Press, 1976).

22. I. M. de Schepper and E. G. D. Cohen, Phys. Lett. 68A:308 (1978); H. van Beijeren, Phys. Lett. 105A:191 (1984); T. R. Kirkpatrick, Phys. Rev. Lett. 53:1735, 2185 (1984).

23. J. J. Erpenbeck and W. W. Wood, Phys. Rev. A 32:412 (1985).

24. J. P. J. Michels and N. J. Trappeniers, Chem. Phys. Lett. 33:195 (1975); M. C. Marchetti and J. W. Dufty, Phys. Rev. A 24:2116 (1981).

25. S. Chapman and T. G. Cowling, The Mathematical Theory of Non-Uniform Gases (Cambridge University Press, Cambridge, 1960).

26. H. van Beijeren, J. Karkheck, and J. V. Sengers, Phys. Rev. A 37:2247 (1988). 